\title{
Estimation of biological parameters of marine organisms using linear and nonlinear acoustic scattering model-based inversion methods
}

\author{
Dezhang Chu, ${ }^{1, a)}$ Gareth L. Lawson, ${ }^{2}$ and Peter H. Wiebe ${ }^{2}$ \\ ${ }^{1}$ Northwest Fisheries Science Center, National Marine Fisheries Service, National Oceanic and Atmospheric \\ Administration, 2725 Montlake Boulevard East, Seattle, Washington 98112, USA \\ ${ }^{2}$ Woods Hole Oceanographic Institution, Woods Hole, Massachusetts 02543, USA
}

(Received 23 July 2015; revised 30 March 2016; accepted 25 April 2016; published online 19 May 2016)

\begin{abstract}
The linear inversion commonly used in fisheries and zooplankton acoustics assumes a constant inversion kernel and ignores the uncertainties associated with the shape and behavior of the scattering targets, as well as other relevant animal parameters. Here, errors of the linear inversion due to uncertainty associated with the inversion kernel are quantified. A scattering model-based nonlinear inversion method is presented that takes into account the nonlinearity of the inverse problem and is able to estimate simultaneously animal abundance and the parameters associated with the scattering model inherent to the kernel. It uses sophisticated scattering models to estimate first, the abundance, and second, the relevant shape and behavioral parameters of the target organisms. Numerical simulations demonstrate that the abundance, size, and behavior (tilt angle) parameters of marine animals (fish or zooplankton) can be accurately inferred from the inversion by using multi-frequency acoustic data. The influence of the singularity and uncertainty in the inversion kernel on the inversion results can be mitigated by examining the singular values for linear inverse problems and employing a non-linear inversion involving a scattering model-based kernel.
\end{abstract}

[http://dx.doi.org/10.1121/1.4948759]

Pages: 2885-2895

\section{INTRODUCTION}

Quantitative information on the spatial and temporal distributions of aquatic organisms is essential to the understanding and management of marine ecosystems. Biological sampling methods such as nets, trawls, and pumps typically have been used to provide direct data (Wiebe and Benfield, 2003). However, these techniques can only provide sparse discrete information and are time consuming, notwithstanding the inherent biases due to sampler avoidance (Kelley, 1976; Wiebe et al., 2013). Acoustic remote sampling techniques can continuously sample a much larger portion of the water column during a given time period than the direct methods. Since the acoustic sampling is indirect, conversion from the measured acoustic intensity and frequency response to abundance and/or biomass is required (Greenlaw, 1979; Lavery et al., 2007; Demer, 2004).

Earlier applications using multi-frequency acoustic measurements to estimate zooplankton size distributions quantitatively can be traced back to the work by McNaught in the late 1960s (McNaught, 1968). Their approach was primarily based on an empirical model of zooplankton target strength obtained by linear regressions between the measured acoustic intensities and co-registered net sampling. A formal theoretical formulation based on the linear inversion theory proposed by Holliday (1977) established a framework for later development of acoustic inversion techniques in zooplankton and fisheries applications. Using the theory of generalized linear inversion, an influential paper by Holliday et al. (1989) reported on the application of the Multi-frequency Acoustic Profiling System to the

${ }^{a)}$ Electronic mail: dezhang.chu@noaa.gov determination of zooplankton size distribution. The system contained 21 discrete frequencies logarithmically distributed between $100 \mathrm{kHz}$ and $10 \mathrm{MHz}$ and was used to estimate the biomass distribution of zooplankton of different groups. The decades subsequent to these initial developments have seen a variety of applications of multi-frequency acoustic inversions to studies of zooplankton (e.g., Lavery et al., 2007; Lawson et al., 2008a; Lawson et al., 2008b; Holliday et al., 2009; Korneliussen et al., 2009; Lebourges-Dhaussy et al., 2009).

The introduction of acoustic scattering models for constructing the inverse kernel matrix required for such inversions and the associated error analysis in terms of the model and measurement errors led to a series of advances in modeling the acoustic scattering by marine organisms (Holliday, 1976; Love, 1977; Foote, 1985; Stanton, 1989; Clay, 1992; Stanton et al., 1993, 1998; Clay and Horne, 1994; Chu et al., 1993; Ye, 1997; Horne and Jech, 1999; Stanton and Chu, 2000; Demer and Conti, 2005; Lawson et al., 2006). The conventional linear inversion technique for estimating zooplankton abundance assumes that the multi-frequency data are independent of, or at least not perfectly dependent on, each other and that the scattering model inherent to the inversion kernel is perfectly known. The effect of uncertainty in the scattering models and associated parameterization on uncertainty in abundance estimates has not been investigated adequately. Although a few publications have studied the uncertainties in estimating acoustically the biomass of marine organisms (Simmonds and MacLennan, 2005; Demer and Hewitt, 1993; Demer, 2004), these works were concentrated on various scattering geometries, notably animal shape and angle of orientation, associated with 
particular acoustic systems under the framework of the sonar equation rather than on the inversion algorithms. Furthermore, deterministic inverse methods accounting for uncertainty in the scattering model parameters and allowing the estimation of these parameters acoustically have seldom been explored in the zooplankton and fisheries literature, although relevant statistical approaches based on the Bayesian method are available from outside disciplines (Tarantola, 2005; Fassler et al., 2009; Menke, 2012; Xiang and Fackler, 2015).

Here we present a systematic approach to a more generalized inversion problem including both linear and nonlinear inversions. The corresponding uncertainty associated with the inversion kernel is investigated, revealing the uncertainties related to the scattering models. The nonlinear inversion presented allows a parametric inversion to find both scatterer abundance and the optimized scattering model and associated parameters. Additional information, including the upper and lower bounds of the animal distribution parameters, biological and acoustic distribution statistics, and the uncertainty constraints, is also integrated into the inversion by means of linear and/or nonlinear programming.

\section{METHODS}

\section{A. Theoretical framework}

In this section, the theoretical background of linear and nonlinear inversions is briefly described including both inversion and the associated uncertainties. Focus is on multifrequency data available from a majority of the acoustic systems currently used in fisheries and zooplankton acoustics.

\section{Linear and nonlinear inversion}

For a multi-frequency acoustic system, the received differential backscattering cross-section per unit volume for the $i$ th frequency can be expressed as (Holliday, 1977)

$$
\sigma_{\text {tot }}^{i}(\zeta)=\sum_{j=1}^{N} n_{j} \sigma_{j}^{i}(\zeta), \quad i=1,2, \ldots M
$$

where $M$ is the number of frequencies, $N$ is the total number of animal groups used in the inversion, $n_{j}$ is the number of animals per unit volume for the $j$ th animal group, and $\sigma_{j}^{i}$ is the differential backscattering cross-section of $j$ th animal group at the $i$ th frequency. $\zeta$ is a feature vector specifying the geometrical (size, shape, and orientation) and physical (density and sound speed contrasts, attenuation, etc.) properties of the animals. Our objective is to determine $n_{j}$ through Eq. (1). The inverse problem is to minimize a cost function, or an objective function,

$$
Q_{d}=\sum_{i=1}^{M}\left\|\sigma_{\text {tot }}^{i}(\zeta)-\sum_{j=1}^{N} n_{j} \sigma_{j}^{i}(\zeta)\right\|_{2}^{2},
$$

where $\|\bullet\|_{2}$ is the $L_{2}$ norm. In matrix form, Eq. (2) can be expressed as

$$
Q_{d}=\left(\mathbf{d}_{\sigma}-\hat{\mathbf{d}}\right)^{T}\left(\mathbf{d}_{\sigma}-\hat{\mathbf{d}}\right)=\left(\mathbf{d}_{\sigma}-\boldsymbol{\sigma n}\right)^{T}\left(\mathbf{d}_{\sigma}-\boldsymbol{\sigma n}\right),
$$

where the superscript " $T$ " stands for matrix transpose. $Q_{d}$ represents a measure of the prediction error, or the mismatch between the measured data $\mathbf{d}_{\sigma}$ and the theoretical prediction $\hat{\mathbf{d}}=\boldsymbol{\sigma} \mathbf{n}$. Note that the kernel $\boldsymbol{\sigma}$ is a function of the feature vector, $\zeta$. For $M>N$ and assuming none of $M$ equations defined in Eq. (1) are correlated, the inversion would be a true over-determined inverse problem and the vector $\mathbf{n}$ can be uniquely determined by minimizing $Q_{d}$ with respect to $\mathbf{n}$ (Menke, 2012; Wunsch, 1996),

$$
\mathbf{n}=\left[\boldsymbol{\sigma}^{T} \boldsymbol{\sigma}\right]^{-1} \boldsymbol{\sigma}^{T} \mathbf{d}_{\sigma}=\boldsymbol{\sigma}^{-g} \mathbf{d}_{\sigma},
$$

where $\boldsymbol{\sigma}^{-g}$ is the generalized inverse of the over-determined problem described by Eq. (1). In many cases, the inverse problem is under-determined, i.e., the number of unknowns $N$ is greater than the number of frequencies $M$, and the cost function will then be constructed based on the concept of $s o$ lution length

$$
Q_{\sigma}=\mathbf{n}^{T} \mathbf{n}
$$

The corresponding generalized inverse can be obtained by minimizing $Q_{\sigma}$ subject to the condition defined by Eq. (1),

$$
\mathbf{n}=\boldsymbol{\sigma}^{T}\left[\boldsymbol{\sigma} \boldsymbol{\sigma}^{T}\right]^{-1} \mathbf{d}_{\sigma}=\boldsymbol{\sigma}^{-g} \mathbf{d}_{\sigma} .
$$

Hence, the solution given by Eq. (6) is also called the minimum length solution. For the special case when $M=N$, the kernel matrix $\boldsymbol{\sigma}$ becomes a square matrix, the inversion becomes an even-determined problem. The generalized inverses given by Eqs. (4) and (6) reduce to $\sigma^{-1}$. The inverse exists and the solution, $\mathbf{n}$, can be uniquely determined if the equations for any $i$ th and $k$ th frequencies with $i \neq k$ are linearly independent of each other.

Sometimes, when we are not sure whether Eq. (1) is under-determined, even-determined, or over-determined, an iterative weighted damped least squares solution can be used to minimize a combination of prediction error $Q_{d}$ and solution length $Q_{\sigma}$ (Menke, 2012; Chu et al., 2001),

$$
\mathbf{n}^{(k+1)}=\mathbf{n}^{(k)}+\left[\lambda \mathbf{W}_{n}+\boldsymbol{\sigma}^{T} \mathbf{W}_{d} \boldsymbol{\sigma}\right]^{-1} \boldsymbol{\sigma}^{T} \mathbf{W}_{d}\left[\mathbf{d}_{\sigma}-\boldsymbol{\sigma} \mathbf{n}^{(k)}\right]
$$

where superscript $k$ denotes the $k$ th iteration. The two weighting functions $\mathbf{W}_{n}$ and $\mathbf{W}_{d}$ describe the overall smoothness or flatness of the model and data structures, respectively. In the inversion applications investigated here, elements of the model and data are independent, hence these two weighting matrices are set to the identity matrix, which leads to the solution from the Levenberg-Marquardt algorithm (Levenberg, 1944; Marquardt, 1963). The constant $\lambda$ is a damping parameter and is chosen to ensure the convergence of the iteration and may vary with the iteration number (Chu et al., 2001). Equation (7) is often used when the number of unknowns is slightly larger than the length of the 
data vector $\mathbf{d}_{\sigma}$ or the inverse is close to singular such as in the case of sediment sound speed tomography (Chu et al., 2001).

Inverse solutions given by Eqs. (4), (6), and (7) are valid for linear inverse problems. For a nonlinear inverse problem, such that some components or elements of the feature vector $\zeta$ are also unknown, the kernel matrix $\boldsymbol{\sigma}$ is no longer a constant. To solve a nonlinear inverse problem, we need to determine $\mathbf{n}$ and $\zeta$ simultaneously. For cases when the nonlinear inverse problem can be transformed to a quasi-linear problem (Chu et al., 2001), Eq. (7) can be modified by introducing a new unknown vector $\tilde{\mathbf{n}}$ (which includes the original unknown vector $\mathbf{n}$ and the feature vector $\zeta$ ) and a new kernel matrix $\tilde{\boldsymbol{\sigma}}$ that will be updated for each iteration,

$$
\begin{aligned}
\tilde{\mathbf{n}}^{(k+1)}= & \tilde{\mathbf{n}}^{(k)}+\left[\lambda \mathbf{W}_{n}+\tilde{\boldsymbol{\sigma}}^{(k)^{T}} \mathbf{W}_{d} \tilde{\boldsymbol{\sigma}}\right]^{-1} \\
& \times \tilde{\boldsymbol{\sigma}}^{(k)^{T}} \mathbf{W}_{d}\left[\mathbf{d}_{\sigma}-\tilde{\boldsymbol{\sigma}}^{(k)} \tilde{\mathbf{n}}^{(k)}\right]
\end{aligned}
$$

where $\tilde{\mathbf{n}}=\left[\mathbf{n}^{T} \Delta \zeta^{T}\right]^{T}$ and $\tilde{\boldsymbol{\sigma}}=[\boldsymbol{\sigma} \partial \hat{\mathbf{d}} / \partial \zeta]$, with $[\partial \hat{\mathbf{d}} / \partial \zeta]_{i j}$ $=\partial \hat{d}_{i} / \partial \zeta_{j}$, where $\hat{\mathbf{d}}=\tilde{\boldsymbol{\sigma}} \tilde{\mathbf{n}}$ is the theoretical prediction (Chu et al., 2001). As for Eq. (7), the two weighting matrices, $\mathbf{W}_{n}$ and $\mathbf{W}_{d}$, are normally identity matrices in our applications. Another way to solve a nonlinear inverse problem is to minimize Eq. (2) directly by solving an implicit function $\mathrm{F}(\mathbf{n}, \boldsymbol{\sigma}$, d) as described by Menke (2012, pp. 140-147) subject to a set of constraints

$$
F_{i}\left(\zeta_{j}\right) \geq 0, \text { for } i=1,2, \ldots, p,
$$

through a linear or nonlinear programming approach (Luenberger and Ye, 2008), where $F_{i}\left(\zeta_{j}\right)$ represents the $i$ th constraint on the $j$ th unknown parameter. For $N+\chi>M+p$, where $\chi$ is the number of elements in $\zeta$, and $p$ is the total number of constraints, the inverse problem is underdetermined and the solution is nonunique. To obtain a reasonable unique solution, the condition $N+\chi \leq M+p$ should be satisfied.

\section{Inversion uncertainty}

The generalized inverse solution to a linear inverse problem given in Eqs. (4) and (6) can also be an approximate solution to a nonlinear inverse problem for which the kernel $\boldsymbol{\sigma}$ is a function of the unknown parameter vector $\zeta$. For small uncertainties in $\mathbf{d}$ resulting from measuring error and $\delta \boldsymbol{\sigma}$ resulting from inaccurate scattering models, the variability or uncertainty of the estimated parameter vector, $\Xi_{m}$ may be expressed as (Wunsch, 1996)

$$
\Xi_{m}=\delta \mathbf{n} \delta \mathbf{n}^{T}
$$

where

$$
\delta \mathbf{n}=\boldsymbol{\sigma}^{-g} \delta \mathbf{d}+\delta \boldsymbol{\sigma}^{-g} \mathbf{d} .
$$

The first term on the right-hand side of Eq. (10b) corresponds to the uncertainty for a pure linear inversion. The second term corresponds to the variability in the kernel and is more complicated to evaluate since it depends on the functional relation between $\boldsymbol{\sigma}$ and $\zeta$. The second term, in general, represents a nonlinear inverse problem and can be evaluated numerically. In addition, the inversion constraints or bounds described in Eq. (9) can affect the uncertainty as well.

Equation (10a) is a $N \times N$ matrix and is conveniently used to evaluate the uncertainties of each individual model parameter, especially when these parameters are related to each other, such as the sound speeds in spatially adjacent elements used in acoustic tomography. Another way to estimate the overall uncertainty of the inversion is to compute the sum of squares of the mismatch between the data and the predictions

$$
\Xi_{\mathrm{m}}^{\Sigma}=\delta \mathbf{n}^{T} \delta \mathbf{n}
$$

$\Xi_{\mathrm{m}}^{\Sigma}$ is a scalar and represents the squared sum of the uncertainty contribution from each individual parameter.

For a pure linear inverse problem, i.e., when the second term in Eq. (10b) is negligible compared with the first term, it is obvious that $\delta \mathbf{n}$ is proportional to the square of the $L_{2}$ norm of the generalized inverse $\boldsymbol{\sigma}^{-g}$. To investigate how the structure of the kernel $\boldsymbol{\sigma}$ affects $\left\|\boldsymbol{\sigma}^{-g}\right\|_{2}$ analytically, we analyze the first term in Eq. (10b) for an over-determined inverse problem. It is well known that any $M \times N$ matrix $\boldsymbol{\sigma}$ with $M>N$ can be decomposed into three multiplicative matrices (Penrose, 1955; Menke, 2012; Wunsch, 1996)

$$
\boldsymbol{\sigma}=\mathbf{U} \boldsymbol{\Lambda} \mathbf{V}^{T}
$$

where $\mathbf{U}$ is a $M \times M$ square matrix, whose columns are orthonormal eigenvectors spanning the data space, and $\mathbf{V}$ is a $N \times N$ square matrix, whose columns are also orthonormal eigenvectors, but span the model or parameter space. Equation (11) is called Singular Value Decomposition (SVD) (Menke, 2012; Wunsch, 1996). The singular value matrix $\boldsymbol{\Lambda}(M \times N)$ consists of an $N \times N$ non-zero diagonal matrix, $\boldsymbol{\Lambda}_{N \times N}$, and a $(M-N) \times N$ zero matrix. The values of the diagonal elements of $\boldsymbol{\Lambda}_{N \times N}$ are non-negative and called singular values. Taking advantage of the orthonormality of the eigenvalue matrices $\mathbf{U}$ and $\mathbf{V}$, i.e., $\mathbf{U}^{\mathrm{T}} \mathbf{U}=\mathbf{I}$ and $\mathbf{V}^{\mathrm{T}} \mathbf{V}=\mathbf{I}$, and using Eq. (4), the generalized inverse for an over-determined linear inverse problem can be expressed as

$$
\boldsymbol{\sigma}^{-g}=\mathbf{V} \boldsymbol{\Lambda}_{N \times N}^{-1} \mathbf{U}_{M \times N}{ }^{T} .
$$

Since $\boldsymbol{\Lambda}_{N \times N}$ is a diagonal matrix, its inverse $\boldsymbol{\Lambda}_{N \times N}^{-1}$ is also a diagonal matrix with its elements being the inverse of the corresponding singular values. For a fixed $\delta \mathbf{d}$ in Eq. (10b), the smaller the product of the singular values, the larger the $\left\|\boldsymbol{\sigma}^{-g}\right\|_{2}$, and the larger the $\delta \mathbf{n}$. If the data are truly independent, the $\boldsymbol{\Lambda}_{N \times N}$ will have $N$ non-zero singular values. However, if any of the data at different frequencies are linearly dependent or correlated, $\boldsymbol{\sigma}$ will become rank deficient and the corresponding singular values of the kernel tend to be zero, resulting in a large $\left\|\boldsymbol{\sigma}^{-g}\right\|_{2}$, and hence make the inverse result unstable. If only $K$ singular values that are greater than a threshold $\varepsilon$ are kept and the rest $(N-K)$ singular values are eliminated, the generalized inverse can then be written as 


$$
\boldsymbol{\sigma}^{-g}=\mathbf{V}_{K} \boldsymbol{\Lambda}_{K \times K}^{-1} \mathbf{U}_{K}^{T}
$$

where the minimum singular value in $\Lambda$ satisfies $\lambda_{\min } \geq \varepsilon$. For $K \geq N$ the solution of Eq. (13) is unique and stable. However, if $K<N$ the inverse problem becomes underdetermined and the predicted model parameters are no longer unique. Despite the fact that we can obtain a "unique" solution given by Eq. (6), which is based on the principle of minimum length, the complete solution to Eq. (1) can be proven to be (Wunsch, 1996)

$$
\tilde{\mathbf{n}}=\mathbf{V}_{K} \boldsymbol{\Lambda}_{K \times K}^{-1} \mathbf{U}_{K}^{T} \mathbf{d}+\mathbf{V}_{0} \boldsymbol{\alpha}
$$

where the vector $\boldsymbol{\alpha}$ is an arbitrary coefficient vector that can take any value without violating the original governing equation, Eq. (1). $\mathbf{V}_{0}$ is the matrix containing eigenvectors that span the null space of the model parameters and satisfies the relations

$$
\begin{aligned}
& \mathbf{V}=\left[\mathbf{V}_{K} \mathbf{V}_{0}\right] \\
& \mathbf{V}_{K}^{T} \mathbf{V}_{0}=0 .
\end{aligned}
$$

$\mathbf{V}_{0}$ behaves like an annihilator in model space to which the data cannot be mapped (Menke, 2012). The uncertainty in the predicted model parameter for a rank-deficient system is then (Wunsch, 1996)

$$
\delta \mathbf{n}=\mathbf{V}_{K} \boldsymbol{\Lambda}_{K \times K}^{-1} \mathbf{U}_{K}^{T} \delta \mathbf{d}+\mathbf{V}_{0} \boldsymbol{\alpha} .
$$

The uncertainty matrices defined in Eqs. (10a) and (10c) are then

$$
\begin{aligned}
\Xi_{m}= & \mathbf{V}_{K} \boldsymbol{\Lambda}_{K \times K}^{-1} \mathbf{U}_{K}^{T}<\delta \mathbf{d} \delta \mathbf{d}^{T}>\mathbf{U}_{k} \boldsymbol{\Lambda}_{K \times K}^{-1} \mathbf{V}_{K}^{T} \\
& +\mathbf{V}_{0}<\boldsymbol{\alpha} \boldsymbol{\alpha}^{T}>\mathbf{V}_{0}^{T}, \\
= & \mathbf{V}_{K} \boldsymbol{\Lambda}_{K \times K}^{-1} \mathbf{U}_{K}^{T} \mathbf{R}_{\delta d} \mathbf{U}_{k} \boldsymbol{\Lambda}_{K \times K}^{-1} \mathbf{V}_{K}^{T}+\mathbf{V}_{0} \mathbf{R}_{\alpha} \mathbf{V}_{0}^{T} \\
\Xi_{m}^{\Sigma}= & \delta \mathbf{d}^{T} \mathbf{U}_{k} \mathbf{U}_{K}^{T} \delta \mathbf{d}+\boldsymbol{\alpha}^{T} \mathbf{V}_{0}^{T} \mathbf{V}_{0} \boldsymbol{\alpha},
\end{aligned}
$$

where the matrices $\mathbf{R}_{\delta d}$ and $\mathbf{R}_{\alpha}$ are covariance matrices of vectors $\delta \mathbf{d}$ and $\boldsymbol{\alpha}$, respectively. Note that $\mathbf{U}_{k} \mathbf{U}_{K}^{T}$ is, in general, not equal to an identity matrix. If the elements of the vector $\delta \mathbf{d}$ are independent of each other, the covariance matrix $\mathbf{R}_{\delta d}$ can be replaced by $\sigma_{d}^{2} \mathbf{I}_{K K}$, where $\sigma_{d}^{2}$ is the variance of $\delta \mathbf{d}$, and Eq. (17a) reduces to

$$
\Xi_{m}=\sigma_{d}^{2} \mathbf{V}_{K} \Lambda_{K \times K}^{-1} \mathbf{V}_{K}^{T}+\mathbf{V}_{0} \mathbf{R}_{\alpha} \mathbf{V}_{0}^{T}
$$

The second term in Eq. (10b) corresponds to the variability of the kernel and, in general, has a nonlinear relationship with the parameter vector $\zeta$ described in Eq. (8). Since in general the analytical form of this term may not be obtainable, we can numerically evaluate the second term.

\section{B. Simulations}

Numerical simulations can provide insight into the inversion problem and help us illustrate how the inversion process works. We therefore consider three examples in fisheries and zooplankton acoustics and investigate uncertainties in the inversion kernel. The first example demonstrates how to assess the validity of the inversion result for a pure linear inverse problem, the second example examines the influence of variability in the inversion kernel on the inversion result for a nonlinear inverse problem, and the third example involves a more complicated nonlinear inversion case that demonstrates the non-uniqueness in nonlinear inversion. It should be noted that the examples are highly hypothetical and convenient, and are chosen to illustrate the principle of the inversion application for fisheries and zooplankton acoustics. Furthermore, in addition to the uncertainties explored here, the uncertainties resulting from sampling errors, i.e., the first term of Eq. (10b) and Eq. (16), can be evaluated analytically or numerically with Monte Carlo simulations (Demer, 2004). All inversion programs are written and implemented in MATLAB utilizing the Optimization Toolbox.

\section{Linear inversion example}

In the first simulation example, we explore the errors associated with a linear inversion problem assuming data from an echosounder with two commonly used frequencies in fisheries acoustics, 38 and $120 \mathrm{kHz}$. We further assume that there are two fish groups mixed in the same acoustically ensonified volume, but with different sizes; this might represent, for example, a region where two distinct size classes of herring are present. The mean length of the first group is $28 \mathrm{~cm}$ with a $10 \%$ standard deviation (s.d.), which is assumed fixed, and the mean length of the second group is varied between 6 and $50 \mathrm{~cm}$, also with $10 \%$ s.d. at each length bin (a constant number of lengths bins $n_{\text {bin }}=30$, is used for each length distribution). The abundance or numerical density of both groups is also kept fixed. Our task is to estimate the abundance (number of animals per unit volume) of each of the two fish groups and to estimate the inversion errors for varying lengths of the second size group, as well as for varying levels of simulated noise that represents measurement errors.

The size distributions or probability density functions of both groups are assumed to be Gaussian. Since the swimbladders dominate the backscattering, the simulations consider the scattering from swimbladders only. For simplicity, but without loss of generality, the synthetic data are generated based on the exact modal series solution for fluid spheres (Anderson, 1950). The target strengths of the two fish groups from the gas-filled fluid sphere model depend strongly on frequency (Fig. 1), with a large peak at the low frequency due to a monopole, or breathing mode, resonance scattering, and a relatively flat frequency response at higher frequencies. In addition, the orientation of the swimbladder is assumed to be horizontal, and hence its influence on the backscatter is ignored. Compared to the theoretical target strength of a $28-\mathrm{cm}$ fish, the target strength curve for the variable-length group will shift to the left for larger fish $(>28 \mathrm{~cm})$ and to the right for smaller fish $(<28 \mathrm{~cm})$. However, since the theoretical curves shown in Fig. 1 are the reduced target strength (RTS), i.e., the target strength normalized by the length of the scattering target, the curve 


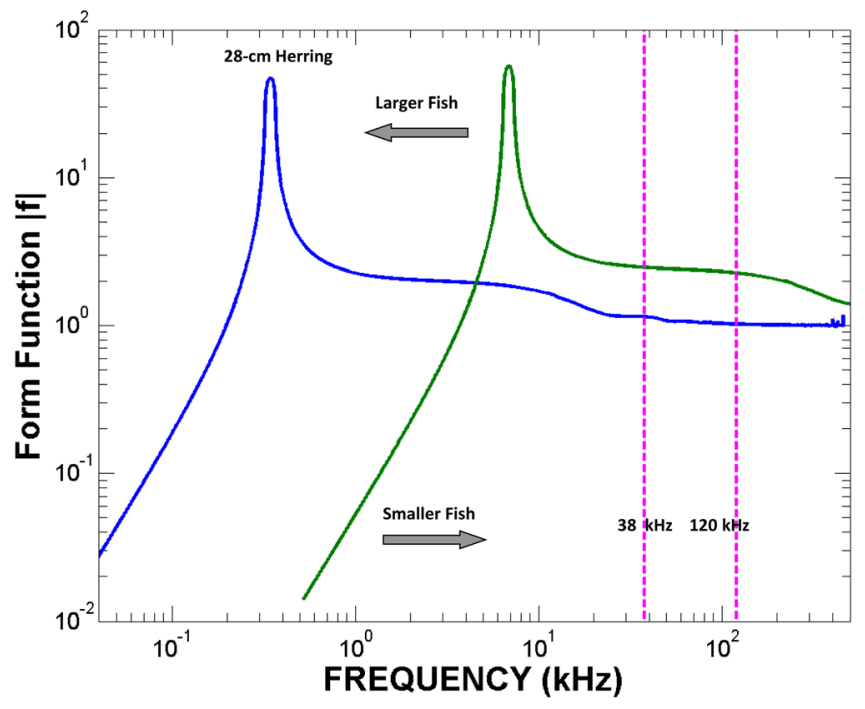

FIG. 1. (Color online) Backscattering form functions as a function of frequency for fish of two different sizes. In simulation example 1 (linear inversion), the size of one group is assumed known $(28 \mathrm{~cm})$, while the other is allowed to vary, with the effect of shifting the theoretical curve left or right for larger or smaller mean sizes, respectively. Since the theoretical curves are the RTS, the curve associated with $6-50 \mathrm{~cm}$ herring is only shifted horizontally relative to the curve labeled $28 \mathrm{~cm}$ herring. The curve on the right (green) corresponds to a fish length of $5.2 \mathrm{~cm}$. The model is based on the fluid sphere model (Anderson, 1950).

associated with $6-50 \mathrm{~cm}$ herring is only shifted horizontally relative to the curve labeled $28 \mathrm{~cm}$ herring. Random noise is also added to the synthesized data with the signal-to-noise (SNR) ratio varying from 6 to $60 \mathrm{~dB}$, which represents the errors in the raw acoustic data. The simulation parameters are tabulated in Table I. Using Eq. (14), the 2-element vector of number density can be computed. The relative error variance is defined as

$$
\varepsilon=\Delta e^{T} \Delta e
$$

where $\Delta e=\left(\tilde{\mathbf{n}}-\mathbf{n}_{\text {true }}\right) /\left\|\mathbf{n}_{\text {true }}\right\|_{2}$. Note that since $M=N=2$, the problem is even- determined and the null space is zero, i.e., $\mathbf{V}_{0}=\mathbf{0}$.

The data vector, $\mathbf{d}$ described in Eq. (3), is the measured volume backscattering strength $\left(S_{v}\right)$ and the kernel $\boldsymbol{\sigma}$ is the scattering model matrix as described in Eq. (1).

TABLE I. Simulation parameters for example, 1, the linear inversion problem (two size classes of fish).

\begin{tabular}{lc}
\hline \hline Parameter & Value \\
\hline Frequencies & $38 \mathrm{kHz}, 120 \mathrm{kHz}$ \\
Fish body lengths & \\
$\quad$ Size group 1 & $28 \mathrm{~cm}$ \\
$\quad$ Size group 2 & $6-50 \mathrm{~cm}$ \\
Ratio of fish body length to swimbladder length & 3.5 \\
Swimbladder aspect ratio & 9.5 \\
SNR & $6-60 \mathrm{~dB}$ \\
Number densities $\left(\right.$ no. $\left./ \mathrm{m}^{3}\right)$ & \\
Size group 1 & 1 \\
Size group 2 & 1.5 \\
\hline \hline
\end{tabular}

\section{Nonlinear inversion case $A$}

The second example investigates the errors resulting from a nonlinear kernel described by the second term of Eq. (10b) and employs the nonlinear inversion (Eq. 8) to estimate simultaneously animal abundance and scattering model parameters inherent to the kernel. In this example, we assume a mono-size fish school with a true mean length of $28 \mathrm{~cm}$, a constant aspect ratio of 10 (ratio of mean length to mean width of swimbladder), a fish numerical density of 2 $\left(\mathbf{n}_{\text {true }}=2\right)$, and a tilt angle of $0^{\circ}$. The synthetic data are generated assuming a 4-frequency echosounder system $(18,38$, $120,200 \mathrm{kHz}$ ) and Eq. (2) is used directly. In the simulation, the swimbladder aspect ratio is varied from 6 to 13 and swimbladder tilt angle varied from $0^{\circ}$ to $15^{\circ}$. In addition, background noise with a $20 \mathrm{~dB}$ SNR is added. The simulation parameters are summarized in Table II. Our objective is to investigate the relative error in estimating the fish number density $(n)$ due to incorrect mean tilt angle $\left(\theta_{\text {tilt }}\right)$ and the mean aspect ratio of the fish swimbladder $\left(r_{\text {asp }}\right)$ used in the inversion processing. The constraints, Eq. (9), for the nonlinear inversion are simply $\theta_{\min } \leq \theta_{\text {tilt }} \leq \theta_{\max }$ for tilt angle and $r_{\min } \leq r_{\text {asp }} \leq r_{\max }$ for aspect ratio, where $\left(\theta_{\min }, \theta_{\max }\right)$ and $\left(r_{\min }, r_{\max }\right)$ were $\left(0^{\circ}, 15^{\circ}\right)$ and $(6,13)$, respectively. These constraints can be expressed as

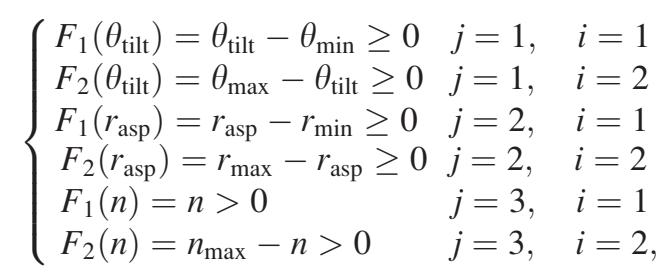

where we can set a realistic upper limit for fish number density $n_{\max }<\infty$. In this example, we set $n_{\max }=100$.

For simplicity as in the linear inversion example (example 1), the backscattering is again assumed to be dominated by that from the swimbladder, i.e., the scattering from fish flesh is ignored. Since the minimum $k a(0.8)$ is much larger than the resonance $k a(0.0132)$, where $k$ is the wavenumber and $a$ is the equivalent spherical radius of the swimbladder, and the tilt angle is less than $15^{\circ}$, the standard method based on the Kirchhoff approximation is used (Foote, 1985).

\section{Nonlinear inversion case $B$}

In this example, hypothetical multi-frequency acoustic data from euphausiids, a group of weakly scattering marine crustaceans, at 5 frequencies commonly used in fisheries and zooplankton acoustics $(18,38,70,120$, and $200 \mathrm{kHz})$ are generated.

Since euphausiids are weakly scattering objects, the Distorted Wave Born Approximation (DWBA) scattering model is used. This model has been successfully used in many previous acoustic studies of euphausiids (Chu et al., 1993; Stanton et al., 1998; Lawson et al., 2006, 2008b). Here, the shape of the animal is modeled as a uniformly bent cylinder (Fig. 2). The "acoustic" length of the equivalent cylinder used in modeling euphausiid scattering is taken as the length from the anterior tip of eye to the end of the sixth 
TABLE II. Simulation parameters for the nonlinear inversion Case A (fish).

\begin{tabular}{lc}
\hline \hline Parameter & Value \\
\hline Frequencies (kHz) & $18,38,120,200 \mathrm{kHz}$ \\
Mean fish body length & $28 \mathrm{~cm}$ \\
Swimbladder (simulation): & Prolate spheroid \\
Shape & $9.3 \mathrm{~cm}$ \\
Length & 10 \\
Aspect ratio & $0^{\circ}$ \\
Tilt angle & \\
Swimbladder (inversion): & Prolate spheroid \\
Shape & $9.3 \mathrm{~cm}$ \\
Length & $6-13$ \\
Aspect ratio & $0^{\circ}-15^{\circ}$ \\
Tilt angle & $20 \mathrm{~dB}$ \\
SNR & 2 \\
Number density (no./m $\left.{ }^{3}\right)$ & 2 \\
\hline \hline
\end{tabular}

abdominal segment. The parameters that we want to estimate are abundance $\left(n\right.$, no. $\left.\mathrm{m}^{-3}\right)$, the mean length $(\bar{L})$, the mean tilt angle $(\bar{\theta})$, and its s.d. $\sigma_{\theta}$. The "true" values of these four parameters used in the simulations are listed in Table III.

The other three geometric parameters required in the DWBA-based scattering model are set as constants during the inversion: $\sigma_{L}$ is the ratio of the s.d. of the animal length to its mean length, $\rho / L$ is the ratio of the radius of curvature of the euphausiid body $(\rho)$ that is assumed to be uniformly bent to the mean length of the euphausiids $(\bar{L})$, and the aspect ratio $(L / W=0.5 L / a$, where $a$ and $W$ are the radius and width of euphausiids, respectively) of the euphausiids. Since the backscattering from euphausiids is relatively insensitive to the parameter $\rho / L$ unless the incidence is close to end-on (Stanton et al., 1993), a value of $\rho / L=3$ is thought to be reasonable and thus is used in our inversion (Chu et al., 1993; Stanton et al., 1993, 1998; Lawson et al., 2006). Although the backscattering cross section is proportional to the square of the aspect ratio of euphausiids, this parameter does not vary too much between individuals for adult euphausiids (Stanton et al., 1998) and, for simplicity, is set to a constant value of $L / a=0.5 L / W=18$. The value is derived from

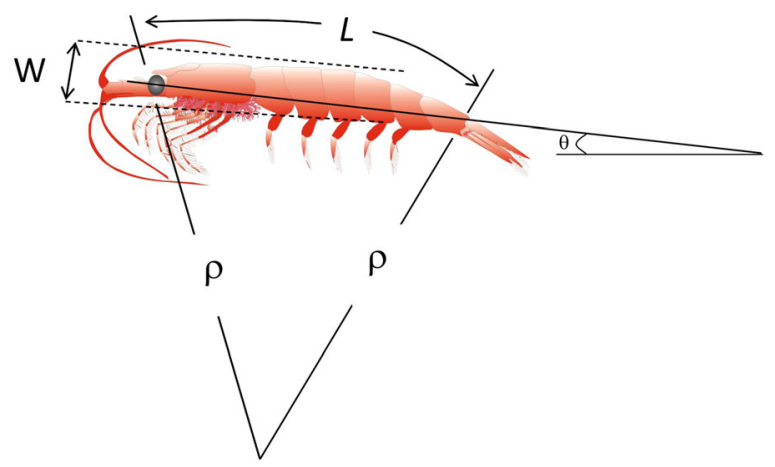

FIG. 2. (Color online) Diagram showing the euphausiid modeling geometry and associated parameters. For the inversions performed here, the unknown parameters of angle of orientation and length distributions are assumed to be Gaussian, where the length distribution is normalized by the mean length. The aspect ratio $L / W$ is assumed to be constant based on previous measurements. The backscattering is insensitive to $\rho / L$ as demonstrated in Stanton et al. (1993) and this parameter is also assumed to be invariant.
TABLE III. Simulation parameters for the nonlinear inversion Case B (euphausiids).

\begin{tabular}{lc}
\hline \hline Parameter & Value \\
\hline Frequencies (kHz) & Uniformly bent \\
Euphausiids (simulation): & 3 \\
Shape & 30 \\
Number density (no./ $\mathrm{m}^{3}$ ) & 0.15 \\
Mean length (mm) & 10 \\
s.d. of length/mean length & $200 \mathrm{kHz}$ \\
Mean tilt angle (deg) & 9 \\
s.d. of mean tilt angle (deg) & 1.04 \\
Aspect ratio & 1.035 \\
Density contrast & 3 \\
Sound speed contrast & \\
Radius of curvature/length & $0.01-10$ \\
Euphausiids (inversion constraints): & $10-50$ \\
Number density (no./m ${ }^{3}$ ) & $0-50$ \\
Mean length (mm) & $0-50$ \\
Mean tilt angle (deg) & $20 \mathrm{~dB}$ \\
s.d. of mean tilt angle (deg) & \\
SNR & \\
\hline \hline
\end{tabular}

measurements of euphausiids sampled in the Gulf of Maine reported in Wiebe et al. (2013), and is also very similar to what we have used before in other modeling studies of elongated scatterers (Chu et al., 1993; Stanton et al., 1993; Stanton et al., 1998). For euphausiids of uni-modal distribution, the parameter $\sigma_{L}$ is about $10 \%$ or less (Foote et al., 1990; Chu and Wiebe, 2005), but could be larger for bimodal length distribution (Lawson et al., 2006, 2008a). In our simulation, a uni-modal length distribution is assumed and this parameter is assigned to 0.15 in order to account for a potential bi-modal length distribution. Two other very important parameters are the sound speed and density contrasts of the euphausiids relative to the surrounding seawater ( $g$ and $h$, respectively). Both of them vary for euphausiids from between 1.007 and 1.04 (Foote et al., 1990; Chu and Wiebe, 2005), and are assigned to $g=1.040$ and $h=1.0350$ in our simulations. These parameters along with other parameters that are necessary to generate the hypothetical mean backscattering cross section per unit volume are summarized in Table III. A stochastic Gaussian random noise is added to the $S_{v}$ with a SNR set to $20 \mathrm{~dB}$.

The nonlinear inversion, based on Eq. (8), is performed to estimate the four unknown parameters: $n,(\bar{\theta}), \sigma_{\theta}$, and $(\bar{L})$. Since the nonlinear inversion method is an iterative process, the initial values for these four parameters are randomly selected within the following bounds, i.e., Eq. (9): $n_{\text {bounds }}$ $=\left[\begin{array}{ll}0.01 & 10\end{array}\right]\left(\right.$ no. $\left./ \mathrm{m}^{3}\right) ; \bar{\theta}_{\text {bounds }}=\left[\begin{array}{ll}0^{\circ} & 50^{\circ}\end{array}\right] ; \sigma_{\theta}$ bounds $=\left[\begin{array}{ll}0^{\circ} & 50^{\circ}\end{array}\right] ;$ $\bar{L}_{\text {bounds }}=\left[\begin{array}{ll}10 & 50\end{array}\right](\mathrm{mm})$. The mathematical expressions for these constraints will have a similar form as Eq. (20).

\section{RESULTS}

\section{A. Linear inversion example}

The first simulation example quantified the errors associated with a linear inversion problem assuming two size 
classes of fish, where the size of the second fish group, as well as SNR, were varied. The simulation relative error variance, $\varepsilon$ defined in Eq. (19), varied strongly as a function of both the mean length of the second fish group and the SNR (Fig. 3a). It is not surprising that in general, the relative error $\varepsilon$ decreases as SNR increases and becomes larger when the mean length of the second group approaches $28 \mathrm{~cm}$, the mean length of the first group. The former is expected since uncertainty in the measurements is decreasing. The latter point is expected since once the sizes of two groups are very close to each other we are not able to distinguish them, resulting in two perfectly correlated equations and producing a singularity in inversion. There are also other spikes around fish lengths 10, 39, and $43 \mathrm{~cm}$, resulting in some ambiguity. To investigate these interpretations quantitatively, the SVD is applied to the kernel matrix given by Eq. (11). In this example, there are two unknowns and two frequencies, where $\boldsymbol{\sigma}$ is a $2 \times 2$ matrix. As a result, we will obtain two singular values. The ratio of the two singular values, or condition number, is plotted as a function of the length of the second fish group in the lower plot of Fig. 3. It is clear that the larger the ratio, the larger the relative error variance in estimating the fish density. The largest peak of the ratio corresponds to the fish length of the second group being $28 \mathrm{~cm}$, which is the same length as that of fish group 1. This is because once the sizes of the two fish groups are equal, the governing equation Eq. (1) becomes singular, or linear dependent, resulting in a zero singular value and causing the ratio to approach infinity. The larger the ratio, the less confident we can be in the estimated number density, and the condition number hence provides a quantitative metric for evaluating inversion uncertainty.

\section{B. Nonlinear inversion case A}

The second simulation example first investigated the relative error in estimating fish density due to incorrect parameterization of the inversion kernel. The relative inversion errors defined in Eq. (19) increased as the swimbladder aspect ratio and tilt angle parameters used in the inversion kernel departed from the true values (Fig. 4, Table II). These two parameters are included in the kernel and are assumed unknown. It can be seen that the inversion error or uncertainty is much more sensitive to the tilt angle, or orientation of the fish, than to the aspect ratio of the swimbladder. The relative error reaches $50 \%$ for using a wrong tilt angle by only about $5^{\circ}$. The minimum error occurs when the tilt angle and aspect ratio used in the inversion match those of the true values.

Our second objective is to estimate simultaneously scattering model parameters inherent to the inversion kernel and fish number density. Since this example is a true overdetermined problem, the kernel is a full rank matrix and the SVD method used in the linear inversion example (example 1) to evaluate the performance of the inversion is ineffective. The degradation of the inversion can be compensated by incorporating the nonlinear inversion method described by Eqs. (8) and (9). The estimated parameters from 100 realizations are
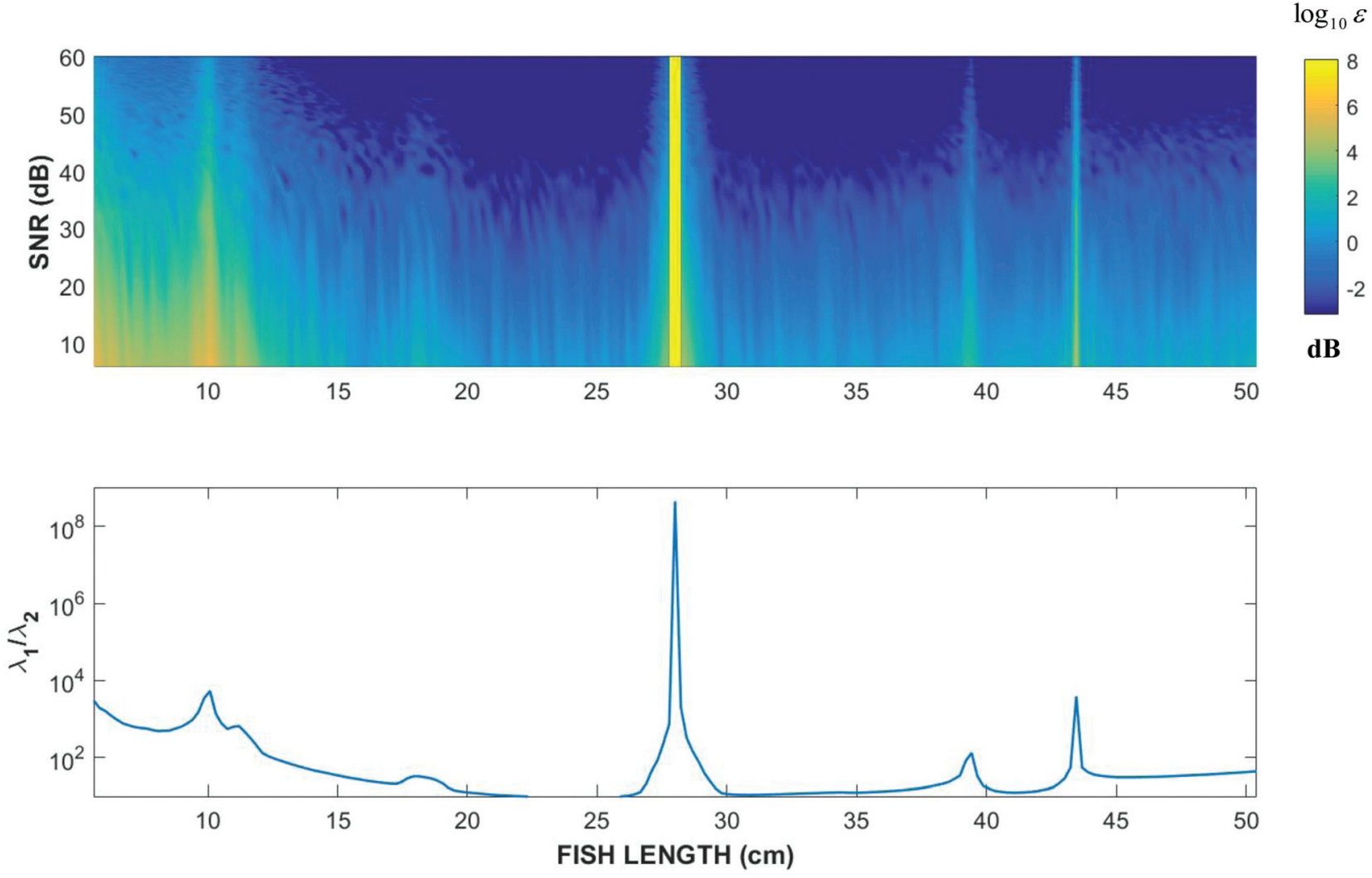

FIG. 3. Results of inversion uncertainty analysis for simulation example 1 (linear inversion) where the fluid sphere model is used to simulate scattering by swimbladders. The upper plot is the inversion error defined by Eq. (19) in log scale $\left(\log _{10} \varepsilon\right)$. The lower plot is the ratio of the two singular values of the inversion kernel matrix $(\boldsymbol{\sigma})$. 


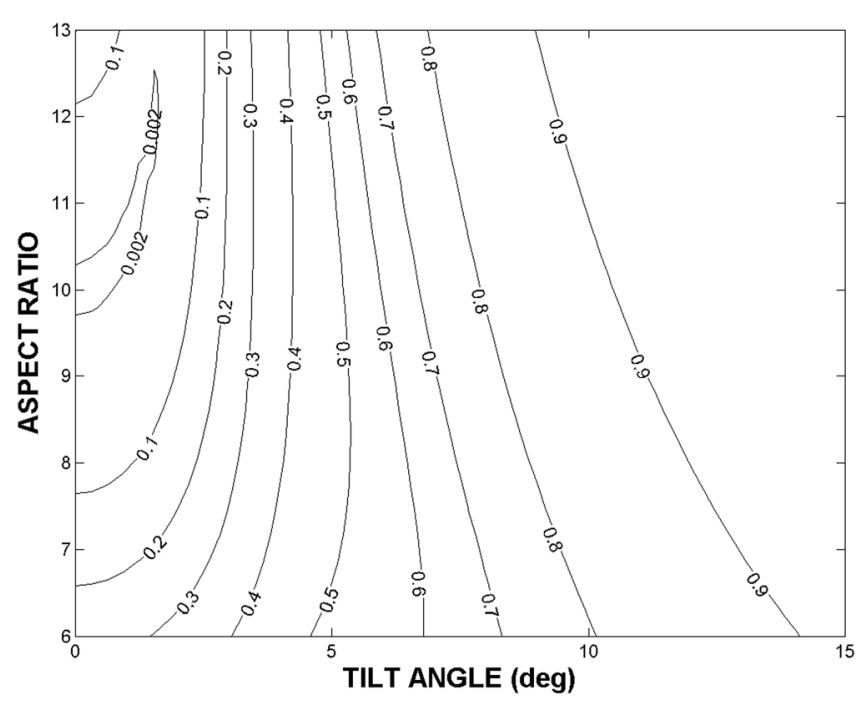

FIG. 4. Contours of inversion uncertainty analysis for nonlinear inversion case A. The true aspect ratio and the tilt angle used in the simulation are $10^{\circ}$ and $0^{\circ}$, respectively. Contours show relative error $(\varepsilon)$ of the estimated numerical density normalized to true density in linear scale. To compare with the quantity used in Fig. 1, the minimum relative error of 0.002 this figure can be evaluated in logarithmic domain as $\log _{10} \quad \varepsilon=\log _{10}$ $0.002=-2.69$.

$\theta_{\text {tilt }}=-0.03^{\circ} \pm 0.04^{\circ}, r_{\text {asp }}=9.98 \pm 0.20$ for tilt angle and aspect ratio, respectively, and $n=2.01 \pm 0.004$. A typical trace of parameter searching history showing the 45 iterations from one realization is illustrated in Fig. 5. The inset shows the estimated fish number density approaching the true pre-assigned simulation value of 2.0 (Table II), where the position of the open square marks the final iteration and the values in parentheses are the final estimated parameters. The number of iterations is determined when the minimum value of $Q_{d}$ defined in Eq. (2) is reached. Here, the relative error term is expressed in a linear case. To compare with the quantity used in Fig. 1, the minimum relative error of 0.002 in Fig. 4 can be evaluated in logarithmic domain as $\log _{10}$ $\varepsilon=\log _{10} 0.002=-2.69$.

\section{Nonlinear inversion case B}

In this example, the four unknown parameters, $n, \bar{\theta}, \sigma_{\theta}$, and $\bar{L}$, are estimated simultaneously using a nonlinear inversion. Three hundred simulated realizations based on the parameters listed in Table III are used to generate the synthetic $S_{v}$ at the 5 frequencies $(18,38,70,120$, and $200 \mathrm{kHz})$ that are commonly used in fisheries acoustics (Table IV). The mean values and the corresponding standard deviations of these parameters inferred from the nonlinear inversion are provided in Table V. Since the number of parameters to be determined from inversion is less than the number of the $S_{v}$ values, it is also an over-determined problem. Largely as a consequence, the agreement between the synthetic data and the theoretical predictions or re-constructed synthetic data using the inferred parameters from inversion is excellent (Fig. 6). The mean difference at each frequency is no more than $0.03 \mathrm{~dB}$ and the overall difference of all five frequencies is about $0.08 \mathrm{~dB}$ (Table IV), which represents a less than $2 \%$ uncertainty in biomass estimate, indicating that local convergence for every realization is reached. The relative error of $\log _{10} \varepsilon$ in this case is -2.59 . The inferred number density $\left(3 \mathrm{~m}^{-3}\right)$ and the mean length $(30 \mathrm{~mm})$ of the euphausiids agree with the assumed true simulation values $\left(3.00 \mathrm{~m}^{-3}\right.$ and $29.97 \mathrm{~mm}$, respectively) very well, while the inferred mean angle of orientation differed from the simulation value by $23 \%$ and the corresponding s.d. agreed reasonably well, but was estimated with a relatively large variance (Table V).

\section{DISCUSSION}

Together, our numerical simulations for the linear and nonlinear inversion serve to highlight the inversion kernel as a key source of uncertainty in multi-frequency acoustic inversions for parameters describing marine organisms and demonstrate the capability of the nonlinear inversion method as a means of simultaneously estimating animal abundance as well as model parameters (e.g., animal size, orientation, etc.) inherent to the kernel.

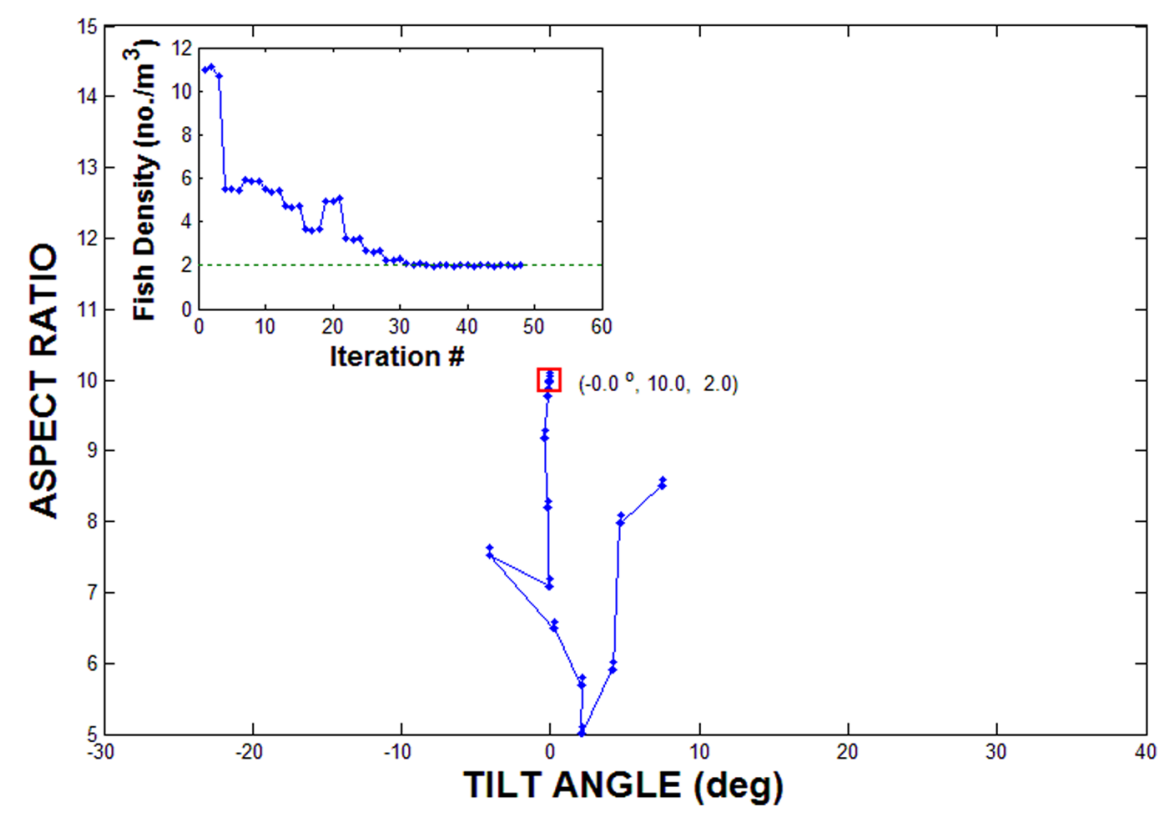

FIG. 5. (Color online) Parameter search trace of the iterative nonlinear inversion case A. The total number of iterations is 45 . The open square marks the location where the iteration stopped. The inferred parameters are $-0.1^{\circ}$ for tilt angle, 10.0 for aspect ratio, and 2.0 for the number density. These parameters are basically identical to the true parameters of $0^{\circ}$ for tilt angle, 10 for aspect ratio, and 2 for fish number density, respectively. The superimposed inset shows the convergence of number density as a function of iteration number. 
TABLE IV. Comparison of the simulated and predicted mean $S_{v}$ values from the nonlinear inversion case B performed on simulated acoustic data at 18,38 , 70,120 , and $200 \mathrm{kHz}$.

\begin{tabular}{|c|c|c|c|}
\hline Frequency (kHz) & Synthetic mean $S_{v}(\mathrm{~dB})$ & Prediction mean $S_{v}(\mathrm{~dB})$ & $<\left|\Delta S_{v}\right|>(\mathrm{dB})$ \\
\hline 18 & -91.22 & -91.24 & 0.02 \\
\hline 38 & -80.35 & -80.38 & 0.03 \\
\hline 70 & -72.79 & -72.81 & 0.02 \\
\hline 120 & -67.97 & -67.98 & 0.01 \\
\hline 200 & -68.47 & -68.47 & 0 \\
\hline Total Error $(\mathrm{dB})=\sum_{i=1}\left|\begin{array}{c}S_{v}^{\text {simulated }}(i) \\
-S_{v}^{\text {pred }}(i)\end{array}\right|$ & - & - & 0.08 \\
\hline
\end{tabular}

The three numerical simulation examples (a linear and two non-linear examples) illustrate the influence of uncertainties in the synthetic data and in the kernel on the relative error in estimating the abundance. Although the inversion itself for the linear inversion example (example 1) is purely a linear inversion, this case demonstrates that the uncertainty or estimate error can be very large even if the SNR is quite favorable $(>40 \mathrm{~dB})$ and the scattering model is correct. It implies an ill-conditioned kernel, i.e., implying the data at two frequencies are correlated and the second datum provides no additional information, which makes the inversion results extremely unstable. The ratio of maximum to minimum singular values or the condition number of the kernel matrix should be taken into account carefully when interpreting the linear inversion results to obtain abundance estimations. As we demonstrate here, the condition number can be used to assess the robustness and stability of the abundance estimations from a linear inversion since it removes the problem due to the singularity of the kernel inverse.

It should be noted that in the first example (the linear inversion example), the two fish groups could be of different species since our scattering model is quite generic. In this example, the scattering model is based on the fluid sphere model (Anderson, 1950). If we had used more sophisticated models such as those given by Love (1977) or Clay and Horne (1994), the specific values of length ranges associated with larger spikes of singular-value ratio could be different, but the general conclusions are expected to be the same.

The nature of inversion uncertainty resulting from an ill-conditioned kernel (larger $\lambda_{1} / \lambda_{2}$ shown in Fig. 3) may also be used to explain the inconsistency between the expected and observed abundance estimates of fish within a length-class reported by Horne and Jech (1999). In their study, a linear inversion was used to predict fish abundance with simulated data from three to five frequencies and the Kirchhoff-ray mode fish backscattering model, which combines the resonance scattering for swimbladder near the

TABLE V. Comparison of the assumed model parameters and inferred model parameters from the nonlinear inversion case B.

\begin{tabular}{lccc}
\hline \hline \multirow{2}{*}{ Parameter } & Assumption & \multicolumn{2}{c}{ Inferred } \\
\cline { 3 - 4 } & & Mean & s.d. \\
\hline$n\left(\mathrm{no} . \mathrm{m}^{3}\right)$ & 3 & 3.00 & 0.08 \\
$\bar{L}(\mathrm{~mm})$ & 30 & 29.97 & 0.01 \\
$\bar{\theta}(\mathrm{deg})$ & 10 & 7.71 & 14.03 \\
$\sigma_{\theta}(\mathrm{deg})$ & 20 & 19.54 & 23.64 \\
\hline \hline
\end{tabular}

resonance frequency, the Kirchoff approximation for swimbladder at higher frequency, and the ray tracing model for fish flesh (Clay and Horne, 1994). Even with data from three to five frequencies and a sophisticated scattering model, their abundance estimates of three length classes in that study were highly variable, indicating that the inversion was not robust and most likely resulting from rank-deficient kernels.

Case A of the nonlinear inversion demonstrates how to solve a nonlinear inverse problem. By combining a scattering model-based nonlinear inversion scheme and the linear programming technique, we are able to estimate the abundance as well as the unknown modeling parameters simultaneously. This approach is similar to a previous application where a nonlinear inversion was applied to field acoustic data for estimating shape and behavioral parameters of cod with a single frequency by using the geometrical and acoustical characteristics of the echo traces (Chu et al., 2003). It is worth noting that the analysis presented in this section is different from the techniques used both by Chu et al. (1993), in which the model parameters were obtained by forward simulations, and by Demer (2004), in which the modeling parameters were not directly estimated from the inversion and were used only for evaluating the uncertainties.

For the case B of the nonlinear inversion, four model parameters are estimated: abundance $(n)$ and mean length $(\bar{L}$,$) ,$ which are the parameters that would be estimated from a

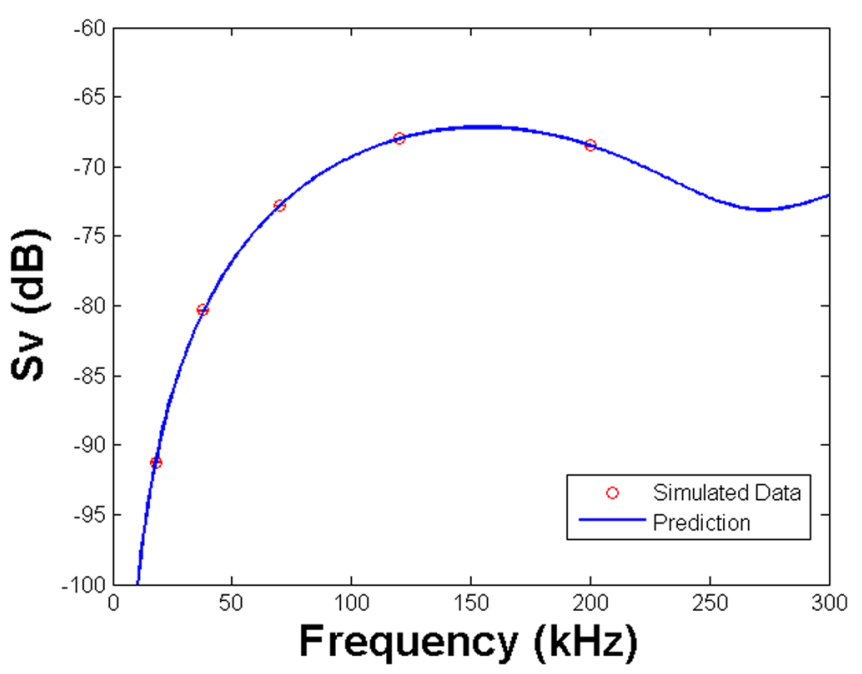

FIG. 6. (Color online) Comparison of the simulated data and the theoretical predictions based on the inferred parameters from the nonlinear inversion for euphausiids (nonlinear inversion case B), i.e., number density, mean, and s.d. of angle of orientation, and mean length. 
traditional linear inversion, and mean tilt angle $(\bar{\theta})$ and its s.d. $\left(\sigma_{\theta}\right)$, which would be assumed to be constants in a linear approach. The two model parameters, $n$ and $\bar{L}$, which are more important and are relatively easy to validate via comparison to direct field sampling methods, are correctly inferred from inversion with very small s.d., indicating their robustness. However, the estimate for $\bar{\theta}$ is $25 \%$ smaller than the true value used in the simulations, but with much larger s.d., a measure of its uncertainty. Although the inferred $\sigma_{\theta}$ from inversion is very close to its assumed value $\left(19.54^{\circ}\right.$ vs $\left.20^{\circ}\right)$, its s.d. is quite large $\left(\sim 24^{\circ}\right)$, indicating large uncertainty. This discrepancy in the estimated parameters concerning tilt angle and the true values used in simulating the data are due to the inherent non-uniqueness of a nonlinear inversion, resulting from the ambiguous nature of the scattering model with respect to $\bar{\theta}$ and $\sigma_{\theta}$ for a uniformly bent cylinder (Stanton et al., 1993). In other words, sometimes, a smaller tilt angle combined with a larger s.d. results in the same target strength as a combination of a larger tilt angle and a smaller s.d.

The non-uniqueness of the two tilt angle related parameters is further demonstrated in Fig. 7. The contours represent the overall mismatch defined as

$$
\Delta_{\Sigma}=\sum_{i=1}^{5}\left|S_{v}^{\text {simu }}\left(f_{i}\right)-S_{v}^{\text {inferred }}\left(f_{i}\right)\right|,
$$

where $f_{i}$ is the $i$ th frequency. There is a minimum region of $\Delta_{\Sigma}$ forming a curved band of $\left[\bar{\theta}, \sigma_{\theta}\right]$ starting approximately from $\left[0^{\circ}, 22.5^{\circ}\right]$, passing through the true values $\left[10^{\circ}, 20^{\circ}\right]$, and then ending at $\left[13.5^{\circ}, 15.5^{\circ}\right]$. Small variation in the data due to the $20 \mathrm{~dB}$ SNR used in the simulation causes the nonlinear inversion to converge to local minimum within the curved band (i.e., 0.2 contour line). However, in reality these two parameters are not easily determined and the exact combination of their values is typically not relevant as long as the target strength values are the same since it will not affect the estimations of abundance and other parameters such as the size of animals.

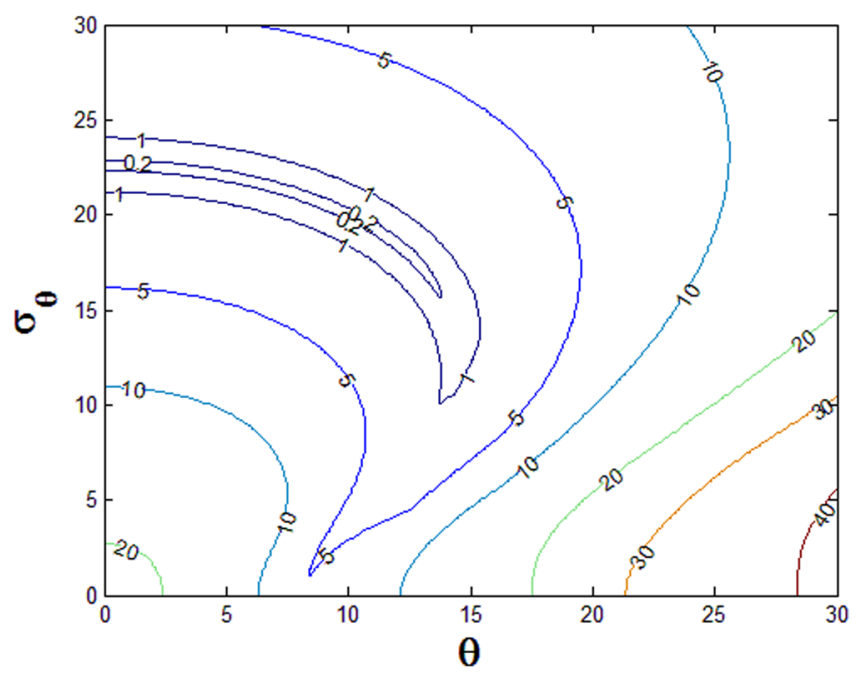

FIG. 7. (Color online) Contours of the overall mismatch between the simulated $S_{v}$ and the predicted $S_{v}$ based on the inferred parameters from nonlinear inversion case B (euphausiids) as a function of tilt angle and its s.d.
Although our examples are highly simplified and idealized compared with most of the inverse problems occurring in fisheries acoustics, the general conclusions raised in the previous paragraphs concerning the usefulness of the nonlinear method should still be valid since the basic underlying physics are the same as in more complicated real-world situations. These numerical simulations also provide stimulus for further investigation of the nonlinear inversion method via application to real-world data. Our findings point to the importance of uncertainty associated with the inversion kernel on inverse estimates of biological parameters, a key point irrespective of the scattering model used.

It is obvious that better and more accurate scattering models will make the inversion results more accurate and the uncertainty related to the scattering model smaller. For the purpose of the simulations presented here, the scattering models are known a priori, and assumed to be representative of the kinds of organisms (fish or zooplankton) being considered. In application to real-world data, however, it becomes very important that the scattering models are correct or at least close to correct. If the physical models are wrong, the inversion can lead to a converged but wrong parameter estimate. In those cases where the correct physical models are not available, empirical models may be used.

\section{SUMMARY}

A theoretical investigation of model-based linear and nonlinear inversion techniques based on multi-frequency acoustic measurements is presented to infer the shape, behavior (specifically, tilt angle), and abundance of naturally-occurring scatterers like fish and zooplankton. Uncertainties for both techniques are investigated with respect to these parameters. For a pure linear inverse problem, where the kernel is perfectly known, the uncertainty of inversion can be evaluated in terms of the singular values or the condition number of the inversion kernel, which is closely related to the fundamental physics of the inversion problem. However, in reality, the parameters required by the scattering models that make up the inversion kernel are frequently not completely known and subsequently will lead to inversion errors. To overcome this, a scattering model-based nonlinear parametric inversion technique can be used to estimate the abundance and the relevant parameters of marine organisms simultaneously. Two numerical examples of this nonlinear technique show its potential to estimate both organism abundance and the parameters that are included in the kernel of the inversion equation. The corresponding uncertainty of the inferred model parameters for a more complicated hypothetical scenario (more unknowns) involving euphausiids is shown to be larger than a simpler scenario involving fish even if both are overdetermined problems, demonstrating the inherent nonuniqueness of the nonlinear inversion. Together, these examples demonstrate the potential of the nonlinear method in fisheries and zooplankton acoustics, and provide stimulus and a basis for applying the method to real-world situations. 


\section{ACKNOWLEDGMENTS}

This work was supported by the National Science Foundation under Grant No. OCE-0928801 and the NOAA National Marine Fisheries Service, Northwest Fisheries Science Center. G.L.L. was partially supported by NOAA Cooperative Agreement Nos. NA09OAR4320129 and NA14OAR4320158 through the NOAA Fisheries Quantitative Ecology and Socioeconomics Training (QUEST) program.

Anderson, V. C. (1950). "Sound scattering from a fluid sphere," J. Acoust. Soc. Am. 22, 426-431 (1950).

Chu, D., Foote, K. G., and Stanton, T. K. (1993). "Further analysis of target strength measurements of Antarctic krill at $38 \mathrm{kHz}$ and $120 \mathrm{kHz}$ : Comparison with deformed cylinder model and inference of orientation distribution," J. Acoust. Soc. Am. 93, 2985-2988.

Chu, D., Jech, J. M., and Lavery, A. (2003). "Inference of shape and behavioral information of individual fish from echo trace analysis," Deep-Sea Res. I 50, 515-527.

Chu D., Tang, D. J., Austin, T. C., and Hinton, A. A. (2001). "Fine-scale acoustic tomographic imaging of shallow water sediments," IEEE Ocean. Eng. 26, 70-83.

Chu, D., and Wiebe, P. H. (2005). "Measurements of acoustic material properties of zooplankton in Antarctic waters," ICES J. Marine Sci. 62, 818-831.

Clay, C. S. (1992). "Composite ray-mode approximation for backscattered sound from gas-filled cylinders and swimbladders," J. Acoust. Soc. Am. 92, 2173-2180.

Clay, C. S., and Horne, J. K. (1994). "Acoustic models of fish: The Atlantic cod (Gadus morhua)," J. Acoust. Soc. Am. 96, 1661-1667.

Demer, D. A. (2004). "An estimate of error for the CCAMLR 2000 survey estimate of krill biomass.," Deep-Sea Res. II 51, 1237-1251 (2004).

Demer, D. A., and Conti, S. G. (2005). "New target-strength model indicates more krill in the Southern Ocean," ICES J. Marine Sci. 62, 25-32.

Demer, D. A., and Hewitt, R. P. (1995). "Bias in acoustic biomass estimates of Euphausia superba due to diel vertical migration," Deep Sea Res. I 42, 455-475.

Fassler, S. M. M., Brierley, A. S., and Fernandes, P. G. (2009). "A Bayesian approach to estimating target strength," ICES J. Marine Sci. 66, 1197-1204.

Foote, K. G. (1985). "Rather-high-frequency sound scattering by swimbladdered fish," J. Acoust. Soc. Am. 78, 688-700.

Foote, K. G., Everson, I., Watkinsa, J. L., and Bone, D. G. (1990). "Target strengths of Antarctic krill (Euphausia superba) at 38 and $120 \mathrm{kHz}$," J. Acoust. Soc. Am. 87, 16-24.

Greenlaw, C. F. (1979). "Acoustical estimation of zooplankton populations," Limnol. Oceanogr. 24, 226-242.

Holliday, D. V. (1976). "Technical report on exploratory development in the application of swimbladder resonance techniques to marine surveys," Tracor Inc., San Diego, CA, Technical Report No. T-76-SD-1101-0, Oct. 19.

Holliday, D. V. (1977). "Extracting bio-physical information from the acoustic signatures of marine organisms," in Oceanic Sound Scattering Prediction, edited by N. R. Andersen and B. J. Zahuranec (Plenum Publishing Corp., New York), pp. 619-624.

Holliday, D. V., Donaghay, P. L., Greenlaw, C. F., Napp, J. M., and Sullivan, J. M. (2009). "High-frequency acoustics and bio-optics in ecosystems research,” ICES J. Marine Sci. 66, 974-980.

Holliday, D. V., Pieper, R. E., and Kleppel, G. S. (1989). "Determination of zooplankton size and distribution with multifrequency acoustic technology," J. du Conseil Int. pour l'Exploration de la Mer 46, 52-61.

Horne, J. K., and Jech, J. M. (1999). "Multi-frequency estimates of fish abundance: Constraints of rather high frequencies," ICES J. Marine Sci. 56, 184-199.

Kelley, J. C. (1976). "Sampling the sea," in The Ecology of the Seas, edited by D. H. Cushing and J. J. Walsh (W. B. Saunders Co., Philadelphia, PA), pp. 351-387.
Korneliussen, R. J., Heggelund, Y., Eliassen, I. K., Oye, O. K., Knutsen, T., and Dalen, J. (2009). "Combining multibeam-sonar and multifrequency echosounder data: Examples of the analysis and imaging of large euphausiid schools," ICES J. Marine Sci. 66, 991-997.

Lavery, A. C., Wiebe, P. H., Stanton, T. K., Lawson, G. L., Benfield, M. C., and Copley, N. J. (2007). "Determining dominant scatterers of sound in mixed zooplankton populations," J. Acoust. Soc. Am. 122, 3304-3326.

Lawson, G. L., Wiebe, P. H., Ashjian, C. J., Chu, D., and Stanton, T. K. (2006). "Improved parameterization of Antarctic krill target strength models," J. Acoust. Soc. Am. 119, 232-242.

Lawson, G. L., Wiebe, P. H., Ashjian, C. J., and Stanton, T. K. (2008b). "Euphausiid distribution along the western Antarctic Peninsula-Part B: Distribution of euphausiid aggregations and biomass, and associations with environmental features," Deep-Sea Res. II 55(3-4), 432-454.

Lawson, G. L., Wiebe, P. H., Stanton, T. K., and Ashjian, C. J. (2008a). "Euphausiid distribution along the western Antarctic peninsula-Part A: Development of robust multi-frequency acoustic techniques to identify euphausiid aggregations and quantify euphausiid size, abundance, and biomass," Deep-Sea Res. Part II 55(3-4), 412-431.

Lebourges-Dhaussy, A., Coetzee, J., Hutchings, L., Roudaut, G., and Nieuwenhuys, C. (2009). "Zooplankton spatial distribution along the South African coast studied by multifrequency acoustics, and its relationships with environmental parameters and anchovy distribution," ICES J. Marine Sci. 66, 1055-1062.

Levenberg, K. (1944). "A method for the solution of certain nonlinear problems in least squares," Qtr. Appl. Math. 2, 164-168.

Love, R. H. (1977). "Target strength of an individual fish at any aspect," J. Acoust. Soc. Am. 62, 1397-1403.

Luenberger, D. G., and Ye, Y. (2008). Linear and Nonlinear Programming, 3rd ed. (Springer, New York), 546 pp.

Marquardt, D. (1963). "An algorithm for least-squares estimation of nonlinear parameters," SIAM J. Appl. Math. 11(2), 431-441.

McNaught, D. C. (1968). "Developments in acoustical plankton sampling," in Proceedings of the 11th Conference Great Lakes Research, pp. 76-84.

Menke, W. (2012). Geophysical Data Analysis: Discrete Inverse Theory, 3rd ed. (Academic Press, Inc., New York), 293 pp.

Penrose, R. A. (1955). "A generalized inverse for matrices," Proc. Cambridge Philos. Soc. 51, 406-413.

Simmonds, E. J., and MacLennan, D. N. (2005). Fisheries Acoustics, 2nd ed. (Blackwell Science, Oxford), pp. 355-379.

Stanton, T. K. (1989). "Sound scattering by cylinders of finite length. III. Deformed cylinders," J. Acoust. Soc. Am. 86, 691-705.

Stanton, T. K., and Chu, D. (2000). "Review and recommendations for the modeling of acoustic scattering by fluid-like elongated zooplankton: Euphausiids and copepods," ICES J. Sci. 57, 793-807.

Stanton, T. K., Chu, D., and Wiebe, P. H. (1998). "Sound scattering by several zooplankton groups. II. Scattering models," J. Acoust. Soc. Am. 103, 236-253.

Stanton, T. K., Chu, D., Wiebe, P. H., and Clay, C. S. (1993). “Average echoes from randomly oriented random-length finite cylinders: Zooplankton models," J. Acoust. Soc. Am. 94, 3463-3472.

Tarantola, A. (2005). Inverse Problem Theory and Methods for Model Parameter Estimation (SIAM, Philadelphia, PA), pp. 41-55.

Wiebe, P. H., and Benfield, M. C. (2003). "From the Hensen net toward four-dimensional biological oceanography," Prog. Oceanogr. 56(1), 7-136.

Wiebe, P. H., Lawson, G. L., Lavery, A. C., Copley, N. C., Horgan, E., and Bradley, A. (2013). "Improved agreement of net and acoustical methods for surveying euphausiids by mitigating avoidance using a net-based LED strobe light system," ICES J. Marine Sci. 70(3), 650-664.

Wunsch, C. (1996). The Ocean Circulation Inverse Problem (Cambridge University Press, New York), pp. 92-211.

Xiang, N., and Fackler, C. (2015). "Objective Bayesian analysis in acoustics," Acoust. Today 11(2), 54-61.

Ye, Z. (1997). "Low-frequency acoustic scattering by gas-filled prolate spheroids in liquids," J. Acoust. Soc. Am. 101, 1945-1952. 